\title{
Erratum to: Splice variants DNMT3B4 and DNMT3B7 overexpression inhibit cell proliferation in $293 \mathrm{~A}$ cell line
}

\author{
Guo Shao $\cdot$ Ran Zhang $\cdot$ Shu Zhang $\cdot$ Shuyuan Jiang $\cdot$ \\ You Liu • Wei Zhang • Yanbo Zhang • Jinping Li • \\ Kerui Gong • Xin-Rong Hu • Shi-Wen Jiang
}

Published online: 21 January 2015

(C) The Society for In Vitro Biology 2015

Erratum to: Publ Vol 49 No 5 pp 386-394 May 2013

DOI 10.1007/s11626-013-9619-z

The Given Name of Keri Gong is incorrect. It should be spelled Kerui.

The online version of the original article can be found at: http://dx.doi.org/10. 1007/s11626-013-9619-z.

G. Shao $\cdot$ S. Zhang $\cdot$ S. Jiang $\cdot$ Y. Liu $\cdot$ W. Zhang

Biomedicine Research Center and Basic Medical College, BaoTou

Medical College, Inner Mongolia, People's Republic of China

G. Shao $\cdot$ J. Li $\cdot$ S.-W. Jiang $(\triangle)$

Hoskins Center, Department of Biological Science,

Mercer University School of Medicine, Savannah, GA, USA

e-mail: jiang_s@mercer.edu

G. Shao $(\bowtie) \cdot$ X.-R. Hu $(\bowtie)$

Department of Pathology, Guangdong Medical College,

Guangdong, People's Republic of China

e-mail: shao.guo.china@gmail.com

e-mail: huxinrong@gdmc.edu.cn

R. Zhang

Peking Union Medical College, TsingHua University, Beijing,

People's Republic of China

Y. Zhang

Department of Neurology, Affiliated Hospital of Tai Shan Medical

College, Taishan, People's Republic of China

K. Gong

Department of Anatomy, University of California, San Francisco,

CA, USA 